\title{
Understanding Reaction Mechanisms in Electrochemistry and Corrosion: Liquid- Cell S/TEM
}

Katherine Jungjohann ${ }^{1}$, Subrahmanyam Goriparti ${ }^{1}$, Claire Chisholm ${ }^{1}$, Bill Mook ${ }^{1}$, Katharine Harrison ${ }^{2}$, Andrew Leenheer ${ }^{3}$, and Kevin Zavadil $^{2}$

1. Center for Integrated Nanotechnologies, Sandia National Laboratories, Albuquerque, USA.

2. Material, Physical \& Chemical Sciences, Sandia National Laboratories, Albuquerque, USA.

3. Microsystems Science \& Technology, Sandia National Laboratories, Albuquerque, USA.

Electrochemical and corrosion studies have greatly benefited from using liquid-cell S/TEM techniques for providing real-time information on the nanoscale mechanisms occurring at solid-liquid interfaces [1]. Within a liquid environment, nanoscale electrodes and metals undergo reactions with the solution creating surface layers and films at the interface. In batteries, these interface layers are known as solidelectrolyte interfaces (SEI). In corrosion experiments, the surface layers are known as scale materials. These systems have related domination of the surface film composition and structure impacting the overall behavior of the electrode and the rate of reaction during corrosion. Therefore, to better understand these material systems and determinant mechanisms, we are investigating using real-time imaging and spectroscopy to characterize these interfaces for initial structural identification, in-situ monitoring of interfacial processes, and post-mortem analysis of electrode/material surfaces.

Electrochemical control within a TEM was pioneered by F. Ross in 2003 using MEMS technology [2] to study electrochemical processes at nanoscale resolution [3-4]. The Center for Integrated Nanotechnologies has successfully designed a microfabricated liquid-cell that can operate with picoampere current control over 10 ultramicroelectrodes while imaging within a TEM, FIG1 [5]. The electrodes are patterned onto a $50 \mathrm{~nm} \mathrm{SiN}$ membrane window, with an even fluid gap around $150 \mathrm{~nm}$. This design allows for multiple experiments to be performed within the same closed cell, where we studied varied current densities and beam conditions under identical environmental conditions. The electrochemical platform is custom patterned using e-beam lithography to deposit working, counter and reference electrodes of customized material composition and volume. In addition, customization of the platform allows for localized heating of the liquid cell in the window region to study elevated temperature influence on electrochemical and corrosion processes [6]. These capabilities are beyond what is currently achievable using a commercial liquid-cell system.

This presentation will cover recent results regarding upgrading our electrochemical TEM discovery platform. We will present on the development of heating and sensing capabilities and their impact on cycling of nanostructured Sn electrodes within carbonate electrolytes for Li-ion batteries. Development of Li metal counter and reference electrodes for cycling nanostructured electrodes within the confined liquid-cell. This capability is important in order to obtain realistic electrochemcial potentials for Li-ion battery systems that accompany high-resolution imaging, FIG2. Low-dose imaging of Li plating/stripping in carbonate electrolytes using the Gatan K2-IS direct electron detection camera for TEM imaging. In addition, experimental results will be provided that highlight the ability to gain insights into corrosion mechanism for steel and shale mineral samples. The breadth of experimental capabilities for in-situ liquid-cell S/TEM are vast, therefore careful characterization of the electron-beam effects for each experiment will be reviewed. This is in effort to define notable conditions that overshadow native mechanisms for these processes [7]. 
References:

[1] F M Ross, Science 350 (2015), p. 1490.

[2] M. J. Williamson et al, Nat. Mater. 2 (2003), p. 532.

[3] A. J. Leenheer et al, ACS Nano 9 (2015), p. 4379.

[4] A. J. Leenheer et al, ACS Nano 10 (2016), p. 5670.

[5] A. J. Leenheer et al, J. Microelectromech. S. 99 (2015), p. 1061.

[6] A. J. Leenheer et al, Microsc. Microanal. 21 (S3) (2015), p. 1293.

[7] This work was performed at the Center for Integrated Nanotechnologies (CINT), an Office of

Science User Facility operated for the U.S. Department of Energy (DOE) Office of Science. Sandia

National Laboratories is a multi-program laboratory managed and operated by Sandia Corporation, a wholly owned subsidiary of Lockheed Martin Corporation, for the U.S. Department of Energy's

National Nuclear Security Administration under contract DE-AC04-94AL85000.

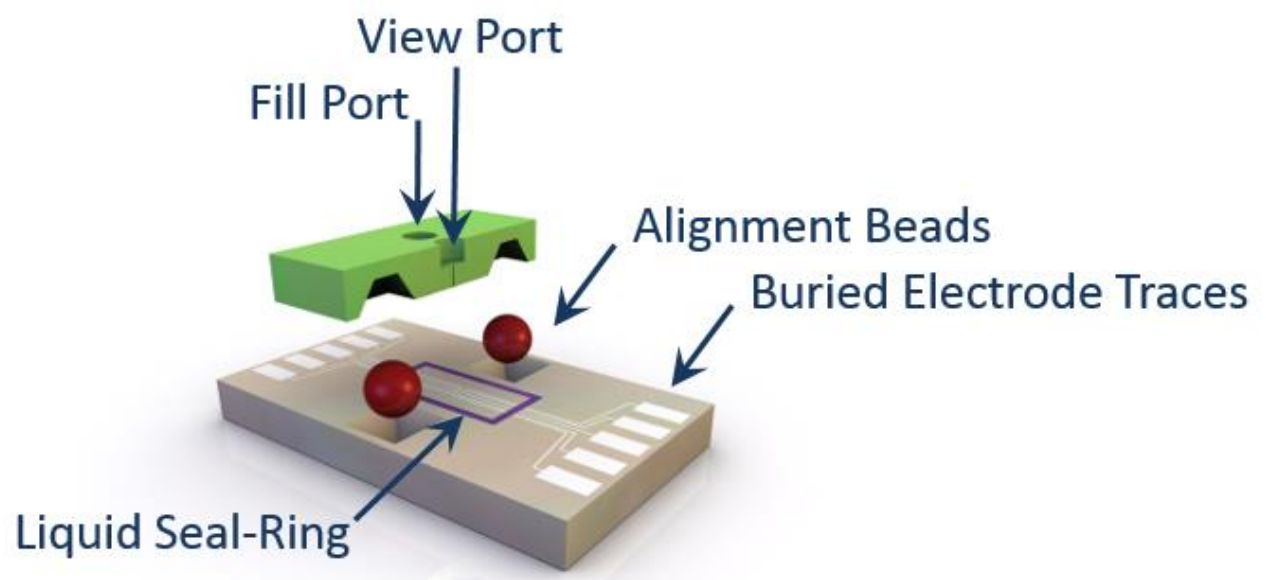

Figure 1. Schematic of electrochemical TEM discovery platform, lid is shown in cross-section to highlight features within the chip. Lid is secured to the base using epoxy after customization of electrodes on the base.

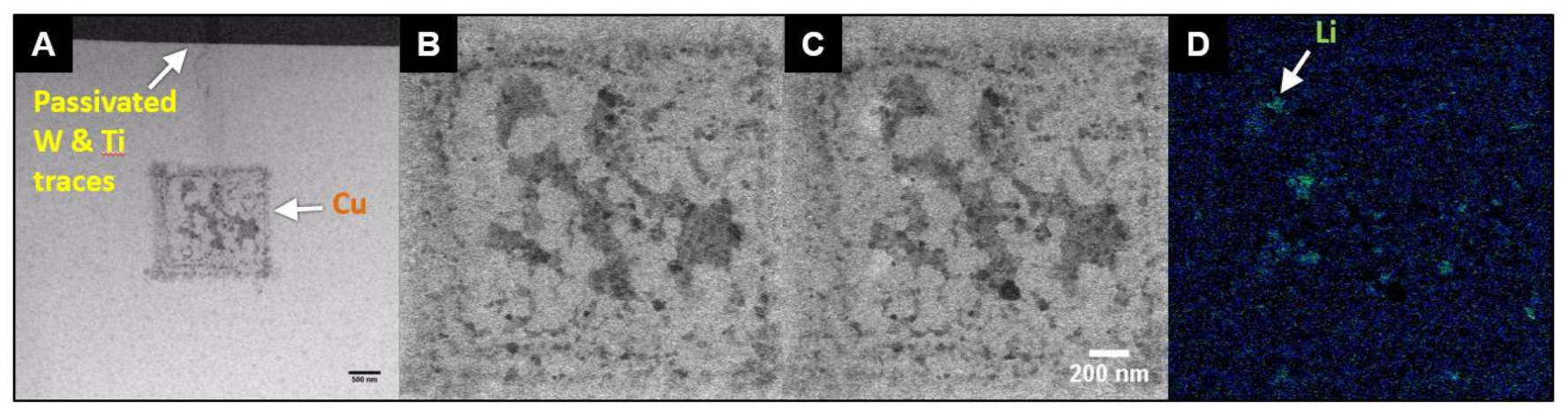

Figure 2. Electrochemical liquid-cell bright-field STEM experiment, detailing the initial nucleation of $\mathrm{Li}$ on copper electrodes from 4M LiFSI in DME electrolyte. A) $\mathrm{Cu}$ electrode patterned from a Ti trace attached to a $\mathrm{W}$ electrode on platform, Ti and $\mathrm{W}$ electrodes were passivated with $33 \mathrm{~nm}$ ALD deposited $\mathrm{Al}_{2} \mathrm{O}_{3}$ (scale bar is $500 \mathrm{~nm}$ ). B) Cu electrode from (a) at higher magnification taken $15 \mathrm{~s}$ after suppling $2.25 \mathrm{~mA} / \mathrm{cm}^{2}$ to deposit Li. C) Cu electrode taken $30 \mathrm{~s}$ after Li plating initiated. D) Subtracted image (b) from (c) to show initial locations of Li deposits on the electrode surface. Image doses of $\sim 1 \mathrm{e}^{-} / \AA^{2}$. 